\title{
Distribución vertical de las aves Dendroica pityophila y Teretistris fernandinae (Passeriformes: Parulidae) en Pinar del Río, Cuba
}

\author{
Alexis Herminio Plasencia Vázquez ${ }^{1}$, Yatsunaris Alonso Torrens² \& Fernando Ramón Hernández \\ Martínez ${ }^{2}$ \\ 1. Museo de Historia Natural "Tranquilino Sandalio de Noda", Martí 202, Esq. Comandante Pinares, Pinar del Río, \\ Cuba, CP 20100. Teléfono: (05348) 779483; aplasencia@mhn.vega.inf.cu, alexpla79@gmail.com \\ 2. Departamento de Forestal, Universidad de Pinar del Río, Martí 270 Final, Pinar del Río, Cuba, CP 20100; \\ yatsunaris@af.upr.edu.cu, fhernandez@af.upr.edu.cu
}

Recibido 13-III-2009. Corregido 01-V-2009. Aceptado 02-VI-2009.

\begin{abstract}
Vertical distribution of the birds Dendroica pityophila and Teretistris fernandinae (Passeriformes: Parulidae) in Pinar del Río, Cuba. The Yellow-Headed Warbler (Teretistris fernandinae) and the Olive-Capped Warbler (Dendroica pityophila) are two bird species that co-exist in Western Cuba. Their spatial distribution has not been studied. This study evaluated their vertical distribution in pine forests during 2007. Six field trips were conducted to the Tibisí pine groves, Minas de Matahambre, Pinar del Río, Cuba, to determine the differential use of forest strata, with the fixed-radio round parcel method. A total of 30 counting points were located in every forest formation. The forest stratum was recorded for each detected bird according to three categories: low stratum ( $0-2 \mathrm{~m}$ over ground), medium stratum $(2-6 \mathrm{~m})$ and high stratum $(6 \mathrm{~m}$ or more). From the 360 counts carried out, the Olive-Capped Warbler reached greater abundances $(3.33 \pm 0.14 \mathrm{ind} / \mathrm{UM})$ than the Yellow-Headed Warbler $(2.32 \pm 024 \mathrm{ind} / \mathrm{UM})$. No abundance differences were found among both forest formations. The Olive-Capped Warbler had the highest frequency in the high stratum and it was observed in the low stratum only during the reproductive season. The Yellow-Headed Warbler had a preference for the low and medium strata, and was seen less frequently in the high stratum. Rev. Biol. Trop. 57 (4): 1263-1269. Epub 2009 December 01.
\end{abstract}

Key words: warblers, spatial segregation, Minas de Matahambre, Pinus tropicalis, Pinus caribaea, Dendroica pityophila, Teretistris fernandinae.

Individuos de diferentes especies pueden diferir en la ocupación de un área, especialmente si el área contiene un mosaico de parches de microhábitats. En el contexto de la teoría de la competencia, estas diferencias pueden ser vistas como facilitadoras de la coexistencia de competidores potenciales en virtud de la segregación espacial, cuando otras características en el uso del hábitat son idénticas (Cody 1974).

A causa de su extrema movilidad las aves son especialmente sensibles a la estratificación vertical en la vegetación. Las investigaciones en esta línea han estado dirigidas al estudio de adaptaciones morfológicas (Dilger 1956), sitios de cría (Turček 1951), conservación del territorio (Kendeigh 1947), abundancia de predadores (Dunlavy 1935) y alimentación (Colquhoun \& Morley 1943, Harrison 1962).

En algunos pinares de la porción más occidental del archipiélago cubano conviven dos especies de bijiritas: la chillina (Teretistris fernandinae), especie endémica de Cuba, y la bijirita del pinar (Dendroica pityophila), que es una especie restringida a los pinares de las provincias de Pinar del Río, Holguín y Guantánamo, que también se encuentra en las Bahamas. Ambas especies tienen caracteres muy similares en su patrón morfológico y en 
sus hábitos alimentarios y en la actualidad se desconoce si utilizan algún tipo de mecanismo para segregarse espacialmente en los pinares.

En el presente trabajo se determinaron los patrones de segregación espacial durante el forrajeo entre la bijirita del pinar y la chillina en los bosques de pinos del Tibisí, Minas de Matahambre, Cuba.

\section{MATERIALES Y MÉTODOS}

La investigación se realizó en la localidad

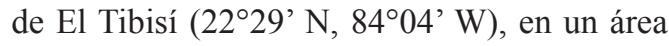
de pinares en la provincia de Pinar del Río, Cuba. Es un área montañosa que se caracteriza por el predominio de pinares, estrechamente relacionados con el bosque de galería y el bosque semideciduo. Los muestreos se realizaron en un pinar natural de pino hembra Pinus tropicalis (Moric) y en una plantación de pino macho Pinus caribaea var. caribaea (Barret $\&$ Golfari) con casi 30 años sin manejo forestal. Ambas formaciones vegetales presentan un sotobosque irregular, con porciones muy tupidas, en ocasiones inaccesibles, y áreas más abiertas en las que predominan las gramíneas.

Los conteos se realizaron en los meses de enero, marzo, mayo, septiembre, noviembre y diciembre del año 2007. Se empleó el método de parcela circular de radio fijo (Hutto et al. 1986) se anotaron todos los individuos vistos u oídos dentro de parcelas de $25 \mathrm{~m}$ de radio, durante $10 \mathrm{~min}$ de observación, de acuerdo a lo sugerido para estudios en bosques tropicales. Se ubicó, marcó y georreferenció, un total de 60 parcelas: 30 en el pinar natural de pino hembra y 30 en la plantación de pino macho, las cuales fueron muestreadas durante todos los meses de trabajo. Los conteos se iniciaban al amanecer y se extendían hasta las 11:00AM. Para cada ave detectada se anotó el estrato del bosque en el cual se encontraban, según tres categorías: estrato bajo ( $0-2 \mathrm{~m}$ de altura), estrato medio (2-6m) y estrato alto ( $6 \mathrm{~m}$ en adelante).

La normalidad de los datos se comprobó por medio de la prueba de Kolmogorov-Smirnov. La evaluación de la estratificación vertical en ambas especies se empleó la prueba de $\mathrm{X}^{2}$, así como para las diferencias en la intensidad de uso, en valores de abundancia proporcional de ambas especies por meses y en la frecuencia de uso y en los valores de abundancia que hacen de los diferentes estratos en ambos tipos de pinares. Para detectar diferencias en los valores de abundancia proporcional de ambas especies, por meses en cada estrato, se empleó una prueba de Kolmogorov-Smirnov de doble distribución. La prueba U de Mann-Whitney se utilizó para detectar diferencias en los valores de abundancia entre las especies.

\section{RESULTADOS}

Las bijiritas fueron relativamente abundantes en ambos pinares. Cuando se comparó el total de individuos (ind) de ambas especies por unidad de muestreo (UM parcela circular) por tipo de pinar, se encuentra que es mayor en $P$. caribaea (abundancia $=2.63 \mathrm{ind} / \mathrm{UM}$ ) que en $P$. tropicalis (abundancia $=2.34 \mathrm{ind} / \mathrm{UM})(\mathrm{Z}=$ $2.16, \mathrm{p}=0.03$ ). De manera general, para el área muestreada se estimaron 2.49ind/UM.

La bijirita del pinar, en general, alcanzó mayores abundancias $(3.33 \pm 0.14 \mathrm{ind} / \mathrm{UM})$ que la chillina $(2.32 \pm 0.24 \mathrm{ind} / \mathrm{UM}) \quad(\mathrm{Z}=4.48$; $\mathrm{p}<0.001)$. Para cada especie no hubo diferencias en los valores de abundancia entre ambas formaciones vegetales (Fig. 1), aunque la potencia de la comparación es muy baja $(<5 \%)$ por el pequeño tamaño de efecto.

En los conteos se encontraron diferencias en la frecuencia en que cada especie de bijirita usa los diferentes estratos de la vegetación $\left(\mathrm{x}^{2}=168.0\right.$, g.l. $\left.=2, \mathrm{p}<0.0001\right)$. Ambas especies usaron todos los estratos, pero la bijirita del pinar tuvo preferencia por el estrato alto mientras que la chillina utilizó con mayor frecuencia los estratos bajo y medio. La prueba de $\mathrm{X}^{2}$ para tendencias, considerando el orden natural de los estratos, da resultados significativos $\left(\mathrm{x}^{2}=165.12 ; \mathrm{g} .1 .=1 ; \mathrm{p}<0.0001\right)$. Cuando se analiza la cantidad de individuos que usaron cada uno de los estratos (intensidad de uso) se detectan diferencias significativas, tanto en el análisis de frecuencia general $\left(\mathrm{X}^{2}=641.9 ; \mathrm{gl}=2\right.$, 

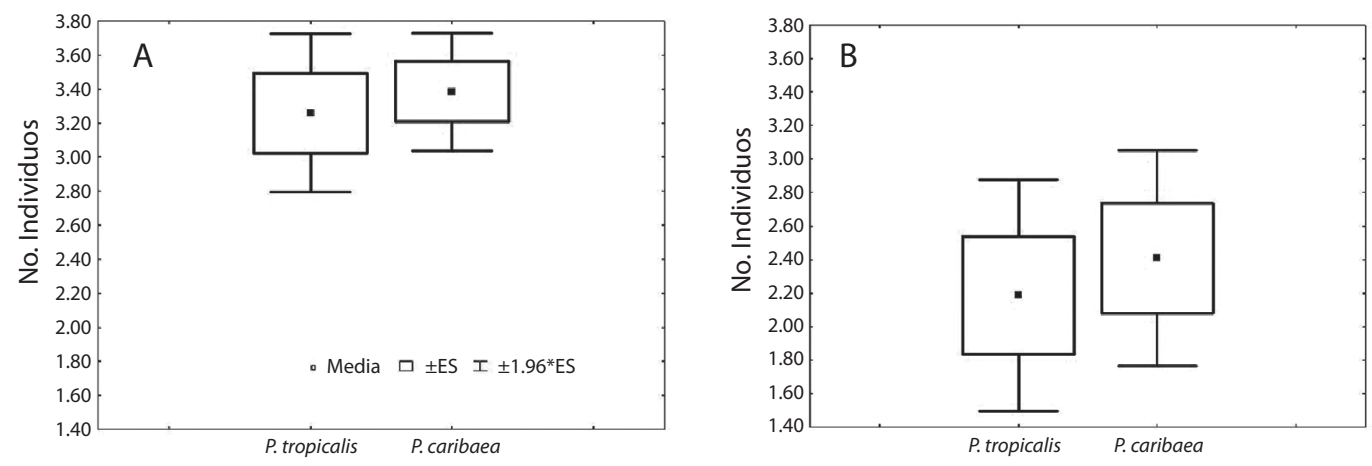

Fig. 1. Valores de abundancia de la bijirita del pinar (D. pityophila) (A) y la chillina (T. fernandinae) (B) en los pinares del Tibisí, Minas de Matahambre, Pinar del Río, según la especie vegetal dominante.

Fig. 1. Abundance values of the olive-capped warbler (D. pityophila) (A) and the yellow-headed warbler (T. fernandinae) (B) according to the dominant pine species in the pinegroves of Tibisí, Minas de Matahambre, Pinar del Río.

$\mathrm{p}<0.001)$ como en el análisis de la tendencia $\left(X^{2}=633.6\right.$; g.l. $\left.=1 ; \mathrm{p}<0.001\right)$.

Al analizar la utilización que hace cada una de las especies de los diferentes estratos por meses se observó que, en ambos casos, existieron diferencias en la frecuencia de uso (Fig. 2). El estrato bajo solo fue usado por la bijirita del pinar en los meses de marzo y mayo, los cuales corresponden a su etapa reproductiva. La chillina utilizó el estrato medio, generalmente, con la misma frecuencia durante todos los meses de muestreo. El estrato bajo no fue visitado en el mes de diciembre. El estrato alto fue el menos empleado por la especie, aunque en el mes de diciembre su valor de frecuencia de uso se elevó.

Al tener en cuenta los valores de abundancia proporcional de ambas especies por meses, se encontraron diferencias significativas entre los estratos alto y bajo, no así para el estrato medio (Fig. 3). Las diferencias son más acentuadas en el caso del estrato alto $(p<0.005)$.

\section{DISCUSIÓN}

La bijirita del pinar se alimenta de insectos en los estratos más altos de las copas de los pinos, donde, además, sitúan sus nidos (Llanes
2002). Esto justifica que en los muestreos realizados apareciera usando con mayor frecuencia el estrato alto, seguido por el estrato medio, en una proporción mucho menor. El estrato bajo solamente fue usado en los meses de marzo y abril, los cuales se corresponden con la etapa reproductiva de la especie (Garrido \& Kirkconnell 2000, Raffaelle et al. 2003). En esta etapa la especie se vuelve más activa en la búsqueda de alimento y se le puede encontrar atrapando insectos en las raíces de los pinos (Garrido 1971) y, probablemente, baje también en busca de materiales para la construcción de los nidos.

Aunque la bijirita del pinar es una especie estricta de pinares, durante la etapa reproductiva se observaron algunos individuos en el bosque de galería adyacente, en las pequeñas galerías dentro de los pinares y en las orillas del río. En esta etapa, al parecer, la especie se vuelve más flexible y explota otros hábitats en busca de recursos que le son necesarios para el desarrollo exitoso de la reproducción, no solo en la parte inferior de los pinares como destaca Garrido (1971) sino que se vale de otras formaciones vegetales.

Las chillinas se observaron asociadas a la vegetación arbustiva de los estratos inferiores 


\section{Bijirita del Pinar}

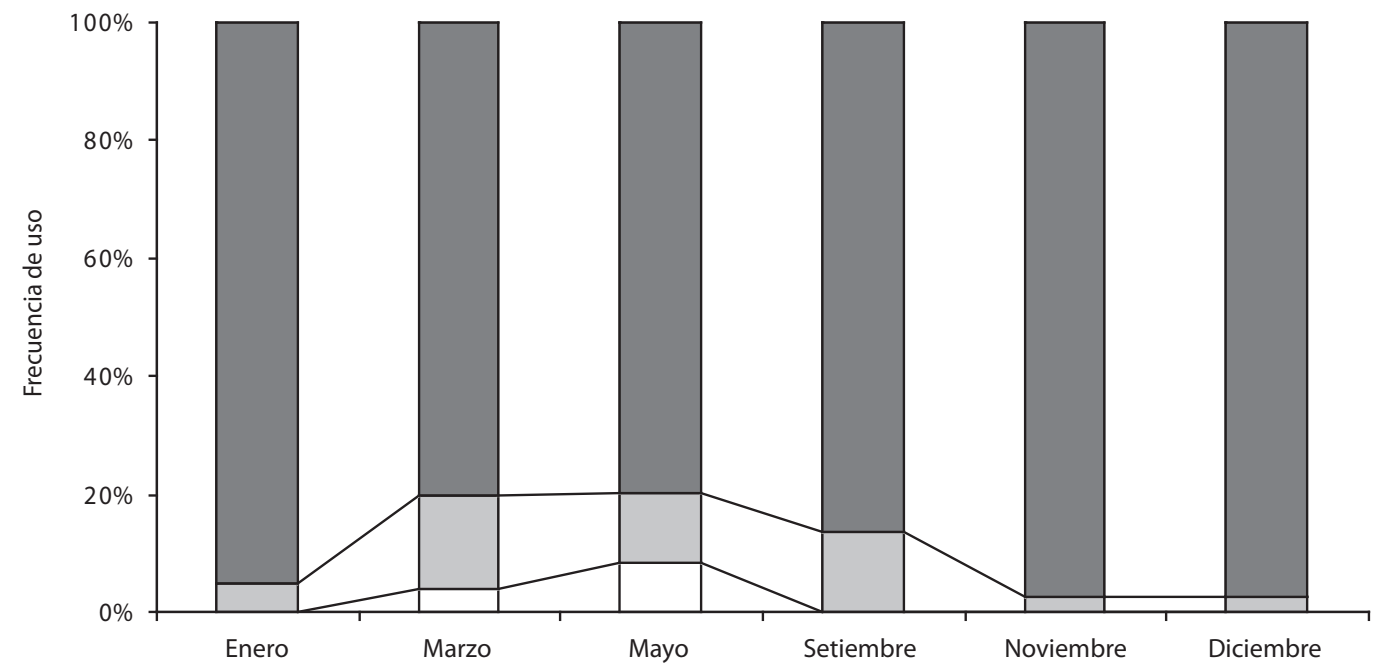

Chillina

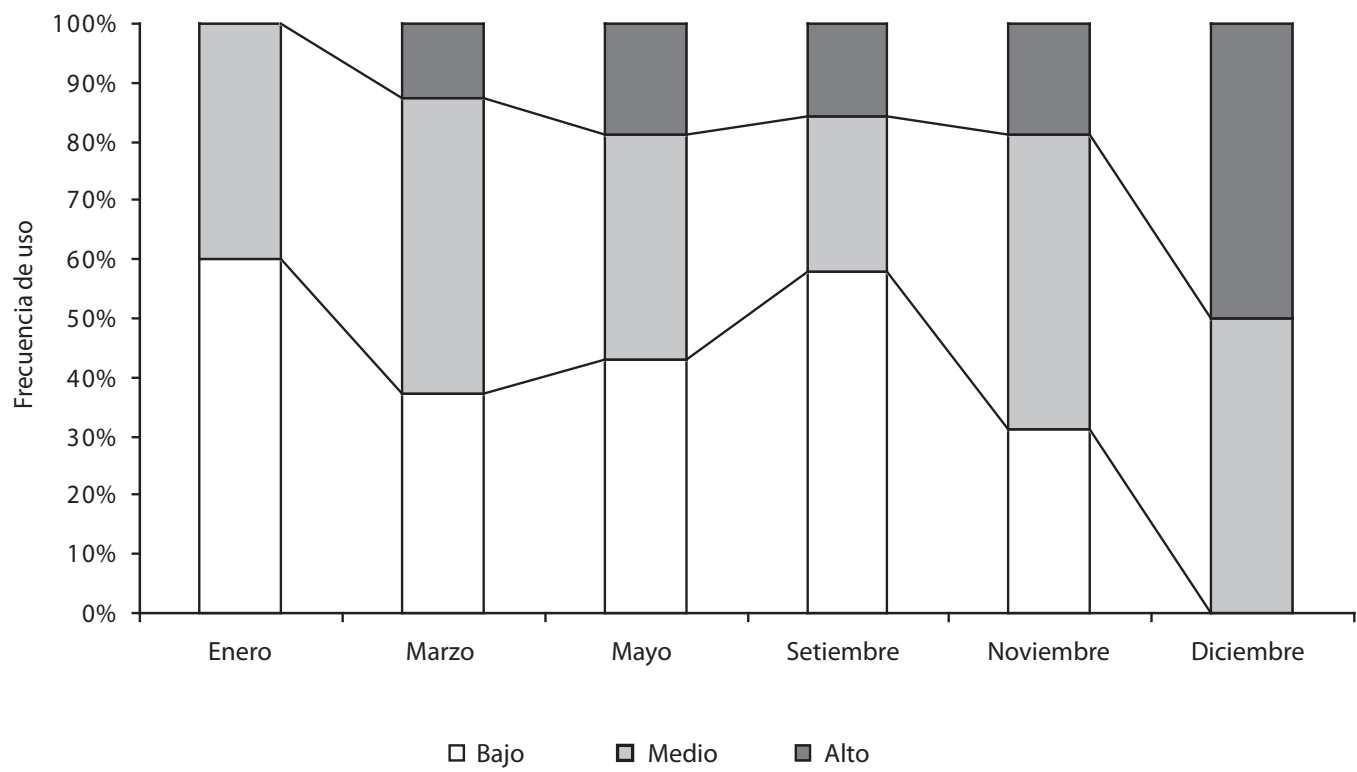

Fig. 2. Diferencias en la frecuencia de uso, por meses, de los estratos del bosque por la bijirita del Pinar (D. pityophila) y la chillina (T. fernandinae) en el Tibisí, Minas de Matahambre, Pinar del Río.

Fig. 2. Differences in the frequency of use of the forest strata by the olive-capped warbler (Dendroica pityophila) and the yellow-headed warbler (Teretistris fernandinae) per month, on Tibisí, Minas de Matahambre, Pinar del Río. 

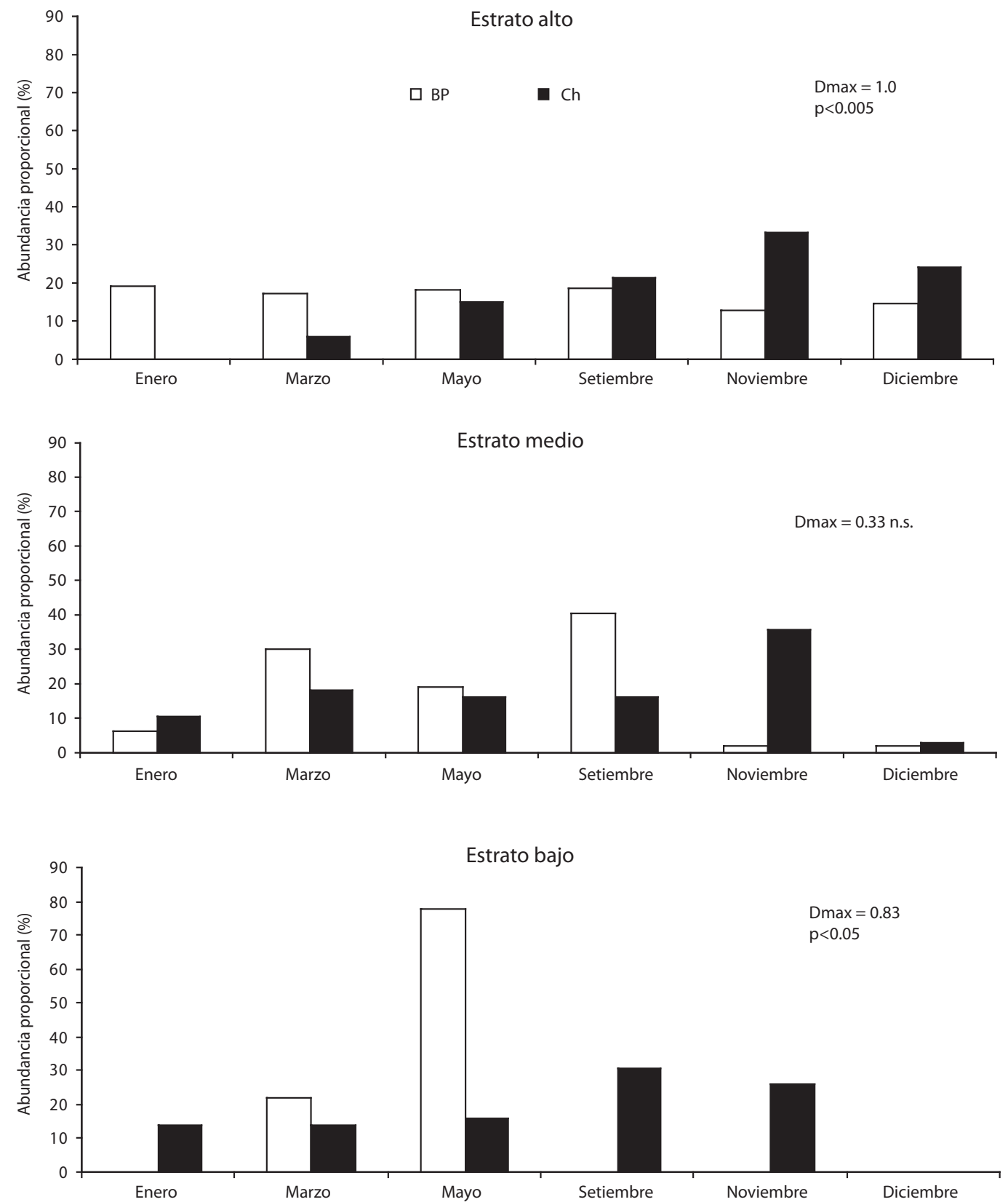

Fig. 3. Variaciones en la abundancia proporcional entre diferentes meses, por estratos de la vegetación, de la Bijirita del Pinar (D. pityophila) y la Chillina (T. fernandinae) en el Tibisí, Minas de Matahambre, Pinar del Río (Resultado de la prueba de Kolmogorov - Smirnov de doble distribución).

Fig. 3. Variations in the proportional abundance during several months, for strata of the vegetation, of the Olive-Capped Warbler (D. pityophila) and the Yellow-Headed Warbler (T. fernandinae) in the Tibisí, Minas de Matahambre, Pinar del Río (Kolmogorov - Smirnov). 
del pinar, así como a los parches de bosque de galería que hay en los mismos. Utilizaron con una frecuencia similar los estratos bajo y medio en los cuales se concentra la mayor parte de la vegetación del sotobosque. En el estrato alto se observaron en menor proporción, y generalmente suben en busca de alimento en los bejucos que crecen a una mayor altura; los cuales son abundantes en varias áreas dentro de los pinares del Tibisí.

Los resultados con relación a la cantidad de individuos, de ambas especies, que usaron cada uno de los estratos fueron similares a los obtenidos para la frecuencia de uso. Para ambas variables, tanto en la bijirita del pinar como en la chillina, las diferencias resultaron estadísticamente significativas, lo cual evidencia una preferencia marcada por un estrato determinado y una segregación espacial de ambas especies en el área. La bijirita del pinar usa con mayor frecuencia y con la mayor cantidad de individuos el estrato alto, mientras que la chillina ocupa el estrato bajo y medio. Estos resultados son similares a los obtenidos por MacArthur (1958) estudiando otras cinco especies de bijiritas (Dendroica sp.) en los bosques de coníferas de Maine; quien demostró que las aves pertenecientes a un mismo gremio alimenticio se segregaban espacialmente durante el forrajeo.

Los valores de abundancia entre las especies mostraron que hay diferencias significativas entre especies. La chillina no es una especie estricta de pinares como la bijirita del pinar, y puede encontrarse en bosques y matorrales desde el nivel del mar hasta grandes elevaciones (Garrido \& Kirkconnell 2000) y también en áreas abiertas (Raffaelle et al. 2003). Por esta razón, no resulta raro que se encuentre en menor número en los pinares ya que en el área de estudio existen otras formaciones vegetales, como el bosque semideciduo, que también utiliza. Rodríguez et al. (1994) hacen alusión a la abundancia de esta especie en diferentes ecosistemas boscosos.

La chillina y la bijirita del pinar en los pinares del Tibisí, Minas de Matahambre, Pinar del Río, se valen de mecanismos que les permiten una coexistencia pacífica. En primer lugar se mueven de forma diferenciada, en frecuencia y número, en los diferentes estratos verticales de la vegetación; lo cual disminuye la posibilidad de interacción entre ambas especies. D. pityophila se localiza generalmente en el estrato alto del bosque sobre los pinos, mientras que $T$. fernandinae se mueve casi todo el tiempo sobre los estratos bajo y medio, donde predomina la vegetación del sotobosque y las bejuqueras, que en ocasiones alcanzan las copas de los árboles.

\section{AGRADECIMIENTOS}

A Elvis Delgado por su apoyo en los viajes de campo. A Dennis Denis Ávila por su ayuda en el procesamiento de los datos y la revisión del documento. A Manuel Díaz Romero por su ayuda en la localización de los puntos de conteo en el área de estudio. A la Empresa Forestal Integral Minas de Matahambre por financiar parte de esta investigación.

\section{RESUMEN}

La bijirita del pinar (Dendroica pityophila) y la chillina (Teretistris fernandinae) son dos especies de ave residentes permanentes en Cuba, que conviven en la región occidental. Se estudió la distribución vertical de estas dos especies en los bosques de pinos. Durante 2007 se realizaron seis muestreos en pinares del Tibisí, Minas de Matahambre Pinar del Río, para determinar el uso diferencial de los estratos del bosque, empleando el método de parcela circular de radio fijo. En los 360 conteos realizados la bijirita del pinar alcanzó mayores abundancias $(3.33 \pm 0.14 \mathrm{ind} / \mathrm{UM})$ que la chillina $(2.32 \pm 0.24 \mathrm{ind} / \mathrm{UM})$. Para cada especie no hubo diferencias en los valores de abundancia entre ambas formaciones vegetales. Se encontraron diferencias en la frecuencia en que cada especie usa los diferentes estratos de la vegetación. La bijirita del pinar utilizó con mayor frecuencia el estrato alto del bosque; en el estrato bajo solo fue observada durante la etapa reproductiva. La chillina tiene preferencia por el estrato bajo y medio, aunque emplea el alto con menor frecuencia.

Palabras claves: bijiritas, segregación espacial, Minas de Matahambre, Pinus tropicalis, Pinus caribaea, Dendroica pityophila, Teretistris fernandinae. 


\section{REFERENCIAS}

Cody, M. L. 1974. Competition and the structure of bird communities. Princeton University, Princeton, Nueva Jersey, EEUU.

Colquhoun, M. K. \& A. Morley. 1943. Vertical zonation in woodland bird communities. J. Anim. Ecol. 12: $75-81$.

Dilger, W. C. 1956. Adaptative modifications and ecological isolating mechanisms in the thrush genera Catharus and Hylocichla. Wilson Bull. 68: 171-199.

Dunlavy, J. C. 1935. Studies on the phyto-vertical distribution of birds. Auk 52: 425-431.

Garrido, O. H. 1971. La bijirita del pinar Dendroica pityophila (Gundlach). Poeyana 84:1-16.

Garrido, O. H. \& A. Kirkconnell. 2000. Field Guide to the Birds of Cuba. Cornell University, Ithaca, Nueva York, EEUU.

Harrison, J. L. 1962. The distribution of feeding habits among animals in a tropical rain forest. J. Anim. Ecol. 31: 53-63.
Hutto, R. L., S. M. P. Letschet \& P. Hendricks. 1986. A fixed-radius point count methods for nonbreeding and breeding season use. Auk 103: 593-602.

Kendeigh, S. C. 1947. Bird population studies in the coniferous forest biome during a spruce budworm outbreak. Biol. Bull. 1: 1-100.

Llanes, A. 2002. Aves de Cuba. Bijiritas y vireos. Ed. Hiram González Alonso, UPC Print, Vaasa, Finlandia.

MacArthur, R. M. 1958. Population ecology of some warblers in northeasters coniferous forest. Ecology 39: 599-619.

Raffaele, H., J. Wiley, O. Garrido, A. Keith \& J. Raffaele. 2003. Birds of the West Indies. Princenton University, Princenton, Nueva Jersey, EEUU.

Rodríguez, D., B. Sánchez, R. Waide y J. Wunderle. 1994. Distribución de aves y conducta social de algunas bijiritas (Passeriformes: Emberizidae) en la Reserva de la Biosfera Sierra del Rosario, Cuba. Cien. Biol. 27: 165-169.

Turček, F. J. 1951. 0 stratifikácii vtáčej populácie lesných biocenóz typu Querceto-Carpinetum na južnom Slovensku. Sylvia 13:71- 86 . 
